\title{
FATORES ASSOCIADOS À INTERRUPÇÃO DE TRATAMENTO ANTI-RETROVIRAL EM ADULTOS COM AIDS. RIO GRANDE DO NORTE, BRASIL, 1999 - 2002
}

Ana Maria de Brito*, Célua landmann Szwarcwald, euclides Ayres de Castilho

Trabalho realizado na Secretaria Estadual de Saúde do Rio Grande do Norte, Natal, RN.

*Correspondência:

Av. Moraes Rego, SN,

Cidade Universitária,

Recife, PE

Cep 50670-900,

anabrito@cpqam.fiocruz.br

\begin{abstract}
RESUMO

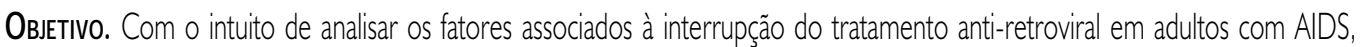
no Rio Grande do Norte, Brasil, realizou-se estudo nas unidades locais de referência no atendimento à AIDS.

Métodos. Tomou-se como critério de não interrupção o comparecimento a pelo menos $80 \%$ das visitas programadas à farmácia para o recebimento das drogas prescritas, por um período de seis meses consecutivos, após a data da prescrição.

Resultados. $O$ estudo compreendeu 498 casos, dos quais 52,4\% já chegaram a um serviço especializado com alguma condição indicativa de imunodeficiência. 0 percentual total de não interrupção foi de $64,1 \%$. Não foi encontrada associação com as variáveis sexo, categoria de exposição e contagem de CD4+, nem com o tipo de esquema anti-retroviral. Os resultados da análise multivariada revelaram associaçōes significativas entre interrupção e início do tratamento após internamento hospitalar, uso de drogas, tratamento psiquiátrico, baixo grau de escolaridade e idade de 25 a 34 anos. Conclusão. Os resultados sugerem que a interrupção do tratamento é um problema crítico nos seis primeiros meses seguintes ao início da terapia anti-retroviral em indivíduos virgens de tratamento, e, especialmente, entre os adultos jovens, com história prévia de tratamento psiquiátrico, que usam ou fizeram uso de drogas licitas ou lícitas até um ano antes de iniciar o tratamento com anti-retrovirais, que iniciam a terapia após internamento hospitalar e têm baixo nível de escolaridade.
\end{abstract}

UNITERMOS: AIDS. Terapia anti-retroviral. Interrupção de tratamento. Adesão.

\section{INTRODUÇÃO}

De uma maneira geral, a literatura médica aponta que a não adesão a cuidados de saúde, em algum grau, é universal, ocorrendo tanto em países ricos como em países pobres, mesmo para doenças que envolvem potenciais riscos de vida. Encontram-se relatos de que pelo menos 40\% a 50\% dos pacientes não têm adesão aos tratamentos de asma, diabetes, hipertensão arterial ou câncer ${ }^{1,2,3}$. Também é importante ressaltar que tal evento pode ocorrer independentemente da classe social, etnia e grau de escolaridade.

Nos vários estudos sobre a adesão a medicamentos, a variável história de doença psiquiátrica é a única a que se mostra consistentemente associada com a falta de adesão. Embora não com a mesma regularidade, outras características, como uso de drogas ilícitas, história de não adesão prévia, e ser morador de rua, têm sido indicadas como importantes fatores associados à falta de adesão 4 .

O critério de adesão em AIDS não tem uniformidade nos diferentes artigos publicados, tanto na literatura nacional como na estrangeira. Os investigadores que estudam a terapia anti-retroviral (ARV) têm adotado vários tipos de métodos e de definições de categorias de adesão. $\mathrm{A}$ maioria dos estudos aceita como ponte de coorte o uso correto de, pelo menos, $80 \%$ das doses prescritas. Não há, porém, confiança suficiente se esse coorte associa-se com parâmetros de melhora clínica e/ou laboratorial $\left.\right|^{5}$.

Os procedimentos utilizados para mensurar a adesão à terapia ARV também diferem entre os estudos. Alguns autores consideram que os métodos diretos de aferiçãa do grau de adesão (questionários ou autorelatos) tendem a superestimar a medida de adesão porque parte dos pacientes não fornece informações corretas sobre as drogas tomadas ${ }^{6}$. Já Hecht et al. (1998) identificaram o auto-relato como mais confiável do que a avaliação médica. Para esses autores, os procedimentos de contagem do número de comprimidos usados não garantem a fidedignidade, uma vez que os comprimidos podem ser descartados e até mesmo os dispositivos eletrônicos para verificar o número de comprimidos usados podem ser manipulados.

Os marcadores biológicos do vírus e as dosagens de metabólitos das drogas são os critérios mais fidedignos para aferir a adesão, mas nem sempre estão disponíveis. Um dos meios de aferi-la indiretamente é pela quantificação da carga viral'. Segundo Carpenter et al. (2000)9 mesmo em estudos controlados, parte importante dos pacientes não atinge o nível desejado de carga viral, menor que 50 cópias do $\mathrm{HIV} / \mathrm{ml}$, sendo que a obtenção de níveis indetectáveis de RNA do HIV, na prática, é bem menor do que em estudos clínicos ${ }^{10,11}$.

Tendo em vista a inexistência de padrões metodológicos, ao se fazer as comparações de resultados obtidos em diferentes estudos para niveis de adesão, é importante que as comparações sempre sejam realizadas à luz do procedimento utilizado, bem como da definição usada para classificação dos níveis de adesão. Adicionalmente, é também importante considerar a população investigada, pois muitos trabaIhos tratam de grupos muito específicos, por exemplo, usuários de drogas injetáveis ou pacientes de um só serviço de saúde. 
Nos poucos trabalhos realizados na população geral, em países desenvolvidos, com o uso de questionamento direto ao paciente e nos quais se considerou a mesma definição de adesão (ou seja, tomar $80 \%$ ou mais de comprimidos prescritos), foram obtidas proporções de $60 \%$, nos EUA ${ }^{12}$, e $75 \%$, na Inglaterra ${ }^{13}$. Investigação realizada em São Paulo mostrou resultado semelhante, com percentual de adesão de 69\% (IC 95\%: 66\%; 71\%).

O atual tratamento da infecção pelo vírus da imunodeficiência humana (HIV) tem contribuído para uma mudança do perfil de morbimortalidade da AIDS, principalmente a partir de 1996, com o uso da terapia anti-retroviral combinada, graças ao advento dos inibidores de protease e análogos não-nucleosídeos ${ }^{14,15}$.

Apesar do indiscutível avanço do tratamento anti-retroviral (ARV), proporcionando um aumento na qualidade de vida dos indivíduos com HIV e AIDS, pela expressiva reduçãa da ocorrência de doenças oportunistas, diminuiç̧ão das internações hospitalares e um aumento significativo no tempo de sobrevida, problemas com esse tipo de terapia têm sido relatados. Os regimes terapêuticos potentes são complexos e apresentam, comumente, problemas de adesão ao tratamento. Os obstáculos para tomada dos medicamentos vão desde um número muito grande de comprimidos por dia - chegando em algumas combinações a se tomar de 10 a 20, em doses divididas, em horários rigorosos, alterações dietéticas ${ }^{14}$ e efeitos tóxicos associados à maioria dos medicamentos ${ }^{16}$.

Além do tratamento específico com os ARV, os indivíduos com AIDS, muitas vezes, necessitam de drogas para tratar co-infecções associadas ao HIV, que implicam, por sua vez, maior número de medicamentos a serem usados, com suas exigências peculiares. Além disso, as possibilidades de interações medicamentosas e reações adversas relacionadas com o uso dessas drogas complicam a terapia ARV potente ${ }^{17}$

A não adesão aos novos medicamentos - anti-retrovirais, em geral, e inibidores de proteases, em particular - tem sido considerada como um dos mais ameaçadores perigos para a efetividade do tratamento, na dimensão individual, e para a disseminação de vírus-resistência, em nível coletivo. Os estudos que têm avaliado fatores de não adesão à terapia ARV apontaram fatores étnicos, uso de drogas e fatores socioeconômicos como relevantes, ${ }^{8,10,18}$. A falta de adesão total ao tratamento pode ser atribuída ainda a problemas psicológicos, culturais e sociais ${ }^{19}$.

O Brasil possui uma rede de diagnóstico e assistência aos enfermos de AIDS no Sistema Único de Saúde, em âmbito nacional, tanto no modelo de atenção tradicional como nos programas mais específicos implementados ao longo dos mais de 15 anos de epidemia. As ações mais específicas incluem outras modalidades de assistência que não somente as internações hospitalares, tais como assistência ambulatorial especializada, hospital-dia e assistência domiciliar, além de um programa de acesso universal aos medicamentos anti-retrovirais que, em 2002, beneficiava mais de 110 mil pessoas $^{20}$.

Embora o sistema de saúde garanta a distribuição e monitoramento dos ARV para todos os casos com indicação de uso desta medicação, inclusive para as situações em que não se caracteriza infecção, mas risco de exposição, como acidentes ocupacionais e recém-nascidos de mães infectadas pelo HIV, não existem ainda mecanismos que possibilitem uma avaliação adequada de adesão à terapia anti-retroviral e, especialmente, para estimar a recusa ao tratamento prescrito. O melhor entendimento sobre os fatores determinantes da adesão, em relação a cada realidade epidêmica, é um dos principais passos para que se possa implementar estratégias de intervenção mais efetivas para o controle da doença.

O presente estudo tem por objetivo analisar os fatores associados à interrupção ao tratamento anti-retroviral, em adultos com AIDS, residentes no Estado do Rio Grande do Norte, bem como estimar o momento em que os indivíduos com AIDS buscam um atendimento específico.

\section{Métodos}

O Rio Grande do Norte, Estado da região Nordeste, com uma população estimada de 2.852.800 habitantes, em 2002 (http:// www.datasus.gov.br), com registro de 1.189 casos acumulados de AIDS, de 1983 a $2002^{20}$, conta com apenas dois serviços de atendimento especializado em AIDS, localizados no município de Natal, capital do Estado, e de Mossoró, município pólo da região Oeste e segundo maior do Estado.

Realizou-se um estudo a partir da revisão das principais bases de dados disponíveis sobre os indivíduos com infecção HIV e AIDS, residentes no Estado do Rio Grande do Norte e atendidos em uma das duas unidades de referência do Estado (Hospital Giselda Trigueiro, em Natal, e Hospital Rafael Fernandes, em Mossoró), que tiveram a primeira prescrição de esquema medicamentoso de ARV entre 10 de janeiro de 1999 e 31 de dezembro de 2001.

Foram incluídos no estudo todos os indivíduos com AIDS, com idade igual ou superior a 13 anos, virgens de tratamento ARV, que tiveram a primeira prescrição de anti-retroviral indicada no período definido no estudo, acompanhados por seis meses, no mínimo. Para permitir o seguimento de todos os indivíduos incluídos, o término da coleta de dados da pesquisa foi em 30 de junho de 2002. De um total de 536 casos elegíveis, foram excluídos os que evoluíram para óbito $(n=17)$ e os que foram transferidos para atendimento em outros Estados $(n=21)$.

Os dados foram coletados em questionário próprio, a partir da revisão de prontuários médicos, de fichas de cadastramento das farmácias de cada unidade de saúde e, para algumas informações não constantes dessas bases, foram consultados os Sistemas de Informação de Agravos de Notificação (SINAN) e de Mortalidade (SIM).

A variável interrupção foi construída a partir da quantidade informada de retirada de medicamentos na farmácia, conforme agendamento, considerando a data de prescrição médica. Tomou-se como critério de não-interrupção a retirada de, pelo menos, $80 \%$ dos medicamentos prescritos, ou seja, os indivíduos que receberam os ARV, pelo menos cinco dos seis meses definidos pelo estudo, segundo o Consenso de Medicação ARV, vigente à época da coleta de dados ${ }^{21,22}$.

As variáveis independentes foram classificadas em dois grupos: sociodemográficas e clínicas. No primeiro grupo, incluiu-se: categoria de exposição ao HIV (heterossexual; homossexual masculino e bissexual masculino); sexo (masculino e feminino); grupo de idade 
BRTTO AM ET AL.

(menor de 25 anos; de 25 a 34 anos; de 35 a 49 anos; 50 anos e mais) município de residência (Natal; Mossoró e interior); e a escolaridade (anos de estudo) (analfabeto ou até três anos; de quatro a oito anos; mais de oito anos).

No segundo grupo, variáveis sobre conhecimento prévio da condição sorológica de positividade do HIV, história de tratamento psiquiátrico prévio à AIDS, uso de drogas lícitas (álcool e medicamentos) ou ilícitas (maconha, crack, cocaína e heroína) até um ano antes da prescrição do ARV, presença de sintomas, sinais ou doenças indicativas de imunodeficiência quando da primeira prescrição de ARV, início da terapia ARV após internamento hospitalar, contagem de linfócitos T CD4+ (inferior a 200 células/mm³; e igual ou superior a 200 células/ $\mathrm{mm}^{3}$ ); quantificação da carga viral no início da terapia ARV (menor do que 4,8 log cópias/ml; e igual ou superior a 4,8 log cópias/ml); tipo de esquema ARV prescrito (duplo; triplo ITRNN - com inibidores de transcriptase reversa não-análogos de nucleosídeos; e triplo IP - com inibidores de protease).

Para cada indivíduo estudado foi construído um indicador binário, com valores zero, correspondendo ao não comparecimento à farmácia, e um correspondendo ao comparecimento. Ao final dos seis meses, obteve-se para cada indivíduo, como soma dos valores do indicador binário, um índice que varia de 0 a 6 . Observa-se que, para efeito de cálculo deste índice, só foram considerados os casos que receberam regularmente a medicação, tendo sido estabelecido, para isso, um prazo de mais 7 e menos 7 dias a partir da data do primeiro comparecimento à farmácia.

Para fins analíticos e de decisão, foram gerados dois grupos: interrupção (índice<5) e não interrupção (índice $\geq 5$ ).

A associação entre os grupos e cada uma das variáveis independentes foi analisada pela estatística - teste do $x^{2}$ (Qui-quadrado), adotandose um nível de significância de 5\%. Posteriormente, foram estimadas odds ratios ajustadas a partir de um modelo de regressão logística, utilizando-se método de escolha das variáveis independentes com base na razão de verossimilhança e níveis de significância de 5\% e 10\% para inclusão e exclusão de variáveis no modelo, respectivamente.

Para estimar o momento em que os indivíduos com AIDS buscam um atendimento específico, procurou-se estabelecer uma associação do conhecimento prévio da condição de soropositividade pelo HIV dos casos com indicação de terapia ARV e a presença de sintomas à primeira consulta a um serviço especializado no atendimento à AIDS, condição esta considerada como oportunidade de atendimento.

\section{Resultados}

\section{ANÁLISE DE FATORES ASSOCIADOS À INTERRUPÇÃO DA TERAPIA ANTI-RETROVIRAL}

O percentual total de não-interrupção à terapia ARV foi de 64, I \%. É importante assinalar que, de um total de 498 casos com indicação de terapia anti-retroviral, incluindo gestantes, cerca de 10,4\% $(n=52)$ dos pacientes nunca foram à farmácia, isto leva a considerar como sendo uma verdadeira recusa.

Para a análise dos fatores associados à interrupção de tratamento foram considerados 426 casos de AIDS com idade igual ou superior a

\begin{tabular}{|c|c|c|c|}
\hline \multicolumn{4}{|c|}{$\begin{array}{c}\text { Tabela } 1 \text { - Proporção de não interrupção dos anti-retrovirais } \\
\text { segundo características sociodemográficas dos casos de AIDS. } \\
\text { Rio Grande do Norte, } 1999 \text { - } 2002\end{array}$} \\
\hline Variável & $\mathbf{N}^{*}$ & $\begin{array}{c}\text { Proporção (\%) de } \\
\text { não interrupção }\end{array}$ & $\begin{array}{c}\text { Valor de } \\
p^{* *}\end{array}$ \\
\hline $\begin{array}{l}\text { Sexo } \\
\text { Masculino } \\
\text { Feminino } \\
\end{array}$ & $\begin{array}{l}298 \\
128 \\
\end{array}$ & $\begin{array}{l}64,1 \\
64,1 \\
\end{array}$ & 0,99 \\
\hline $\begin{array}{l}\text { Faixa etária (anos) } \\
<25 \\
25-34 \\
35-49 \\
50 \text { e }+\end{array}$ & $\begin{array}{l}45 \\
140 \\
185 \\
56\end{array}$ & $\begin{array}{l}71,1 \\
54,3 \\
67,0 \\
73,2\end{array}$ & 0,023 \\
\hline $\begin{array}{l}\text { Categoria de exposição } \\
\text { Heterossexual } \\
\text { Homossexual } \\
\text { Bissexual }\end{array}$ & $\begin{array}{l}162 \\
118 \\
104\end{array}$ & $\begin{array}{l}63,6 \\
60,2 \\
69,2 \\
\end{array}$ & 0,368 \\
\hline $\begin{array}{l}\text { Local de residência } \\
\text { Natal } \\
\text { Mossoró } \\
\text { Interior } \\
\end{array}$ & $\begin{array}{l}278 \\
43 \\
101\end{array}$ & $\begin{array}{l}64,0 \\
67,4 \\
61,4 \\
\end{array}$ & 0,776 \\
\hline $\begin{array}{l}\text { Escolaridade (anosde estu } \\
\text { Analfabeto ou até } 3 \text { anos } \\
\text { De } 4 \text { a } 8 \text { anos } \\
\text { Mais de } 8 \text { anos }\end{array}$ & $\begin{array}{l}170 \\
97 \\
144\end{array}$ & $\begin{array}{l}55,9 \\
68,0 \\
71,5\end{array}$ & 0,011 \\
\hline Total & 426 & 64,1 & \\
\hline
\end{tabular}

* A diferença entre o total geral e 0 total para cada variável deve-se às informaçôes ignoradas

** Nivel de significância descritivo do teste de homogeneidade das proporções de não nterrupção

13 anos, virgens de ARV, com indicação de uso de anti-retrovirais entre $1^{\circ}$ de janeiro de 1999 e 31 de dezembro de 2001 , sendo 298 (70\%) do sexo masculino.

As Tabelas I e 2 apresentam as proporções de não interrupção segundo categorias selecionadas das variáveis sociodemográficas e clínicas. Os resultados dos testes estatísticos indicam que a interrupção não está associada ao sexo, categoria de exposição, local de residência, conhecimento prévio da situação sorológica de $\mathrm{HIV}+$, presença de sintomas no início da terapia ARV, nível de CD4+ e carga viral, nem ao tipo de esquema ARV prescrito.

Entre as características sociodemográficas, a hipótese de homogeneidade das proporções foi rejeitada para grupo etário e escolaridade. A proporçãa de interrupção foi significativamente mais alta em adultos jovens, na faixa etária de 25 a 34 anos, quando comparados com adolescentes e jovens (com menos de 25 anos) e os mais idosos, sendo aproximadamente $20 \%$ mais alta do que a dos indivíduos com 50 anos e mais. Outra característica sociodemográfica associada com a interrupção foi a escolaridade, com proporção de indivíduos com interrupção no grupo de baixa escolaridade aproximadamente $22 \%$ mais alta do que a dos indivíduos com nível de escolaridade médio ou superior (Tabela 3).

Entre as variáveis clínicas, as que se associaram significativamente à interrupção foram: história de tratamento psiquiátrico prévio à AIDS; uso de drogas e início da terapia ARV após 


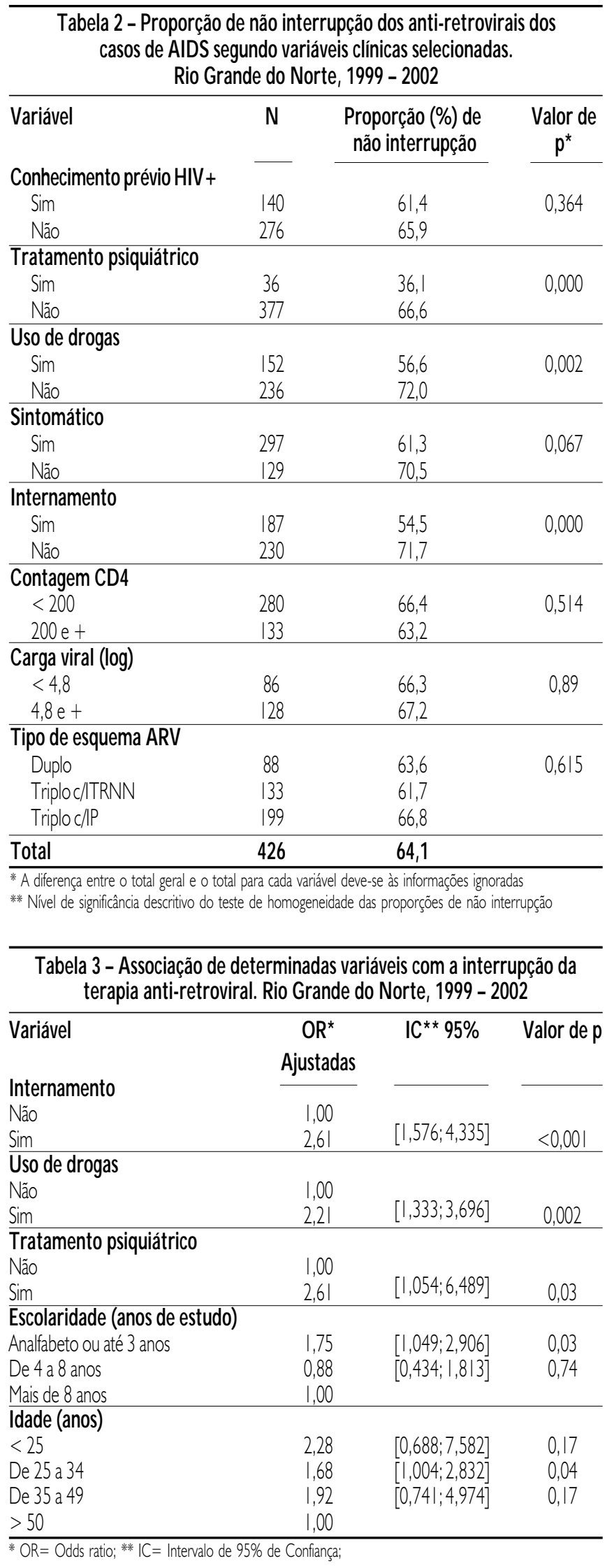

\begin{tabular}{|c|c|c|c|c|c|}
\hline $\begin{array}{l}\text { Conhecimento } \\
\text { prévio HIV }\end{array}$ & № & $\begin{array}{l}\text { Proporção(\%) } \\
\text { com sintomas }\end{array}$ & $\begin{array}{l}\text { Razão de } \\
\text { prevalência }\end{array}$ & IC 95\% & Valor de $\mathrm{P}$ \\
\hline 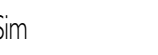 & $\overline{162}$ & 39,4 & 1,50 & {$[1,22-1,86]$} & $<0,00 \mid$ \\
\hline Vão & 308 & 59,4 & & & \\
\hline Total & 470 & 52,4 & - & - & - \\
\hline
\end{tabular}

internamento. A interrupção foi quase duas vezes mais alta em indivíduos com história psiquiátrica quando comparados com os que não tinham transtorno psiquiátrico prévio ao diagnóstico de HIV ou AIDS. O uso de drogas lícitas ou ilícitas mostrou-se significativamente associado com maior interrupção, com uma proporção de interrupção entre os indivíduos com história de uso de drogas (até um ano antes de iniciar a terapia ARV) 21\% mais alta em relação aos que não referem uso de drogas. Da mesma forma, a proporção de interrupção no grupo dos que iniciaram a terapia ARV após internamento foi aproximadamente $24 \%$ mais alta do que os indivíduos que não tinham registro de internamento hospitalar.

Para ajuste do modelo logístico, foram incluídas todas as variáveis sociodemográficas e clínicas, exceto a quantificação da carga viral devido ao grande número de casos sem informação (50,2\%). A Tabela 3 apresenta os resultados da análise multivariada. Associações significativas com a interrupção persistiram para internamento hospitalar, uso de drogas lícitas ou ilícitas, história de tratamento psiquiátrico, baixo nível de escolaridade e idade de 25 a 34 anos.

As variáveis que mais contribuíram para explicar a interrupção foram: ter iniciado a terapia ARV após internamento hospitalar; uso de drogas; e história de tratamento psiquiátrico, sendo a OR ajustada superior a dois, em relação ao grupo de comparação.

\section{Análise de oportunidade de atendimento}

Análise da associação do conhecimento prévio da condição de soropositividade pelo HIV dos casos com indicação de terapia ARV e a presença de sintomas à primeira consulta a um serviço especializado no atendimento à AIDS, condição denominada de oportunidade de atendimento, pode ser realizada mediante apreciação dos dados dispostos na Tabela 4. Os achados revelam que mais da metade dos casos só tomou conhecimento da sua infecção após consulta a um serviço especializado. Os resultados indicam, igualmente, que os indivíduos que não tinham conhecimento prévio da infecção pelo HIV apresentaram 50\% mais chance de ter sintomas à primeira consulta ao serviço especializado do que aqueles que conheciam a condição $(R P=1,50$; $\mathrm{p}<0,001)$.

Dos 498 casos que tiveram indicação de iniciar a terapia antiretroviral, segundo sexo, e, entre as mulheres, se gestante ou não gestante, obteve-se um percentual global de recusa ao tratamento de $10,4 \%(n=52)$ nos seis primeiros meses de seguimento após a primeira prescrição. O resultado de $28,6 \%$ de recusa entre as gestantes é bem superior ao observado para as não gestantes $(6,6 \%)(R P=4,35$; 


\begin{tabular}{|c|c|c|c|c|}
\hline \multicolumn{5}{|c|}{$\begin{array}{c}\text { Tabela } 5 \text { - Distribuição dos casos de recusa da terapia anti-retroviral } \\
\text { segundo sexo e condição gestacional das mulheres. Rio Grande do } \\
\text { Norte, } 1999 \text { - } 2002\end{array}$} \\
\hline \multicolumn{2}{|c|}{ Variável } & N & Proporção (\%) de recusa & Valor de $p$ \\
\hline \multirow[t]{2}{*}{ Sexo } & Masculino & $\overline{333}$ & 10,5 & 0,932 \\
\hline & Feminino & 165 & 10,3 & \\
\hline \multirow{2}{*}{$\begin{array}{l}\text { Estado } \\
\text { gestacional }\end{array}$} & Sim & 28 & 28,6 & \\
\hline & Não & 137 & 6,6 & $0,002 *$ \\
\hline Total & & 498 & 10,4 & - \\
\hline
\end{tabular}

$p<0,002)$ e para os homens ( $10,5 \%)$. A análise dos dados, incluindo as gestantes no grupo de mulheres, não revela diferenças estatísticas de recusa entre os sexos (Tabela 5).

\section{Discussão}

A necessidade da adesão aos esquemas anti-HIV tem sido enfatizada em vários trabalhos sobre terapia anti-retroviral $\left.\right|^{9}$. Teoricamente, seria necessária uma taxa de adesão de mais de $95 \%$ para a obtenção de resultados ótimos da terapia anti-HIV. Os achados do presente estudo indicam um percentual de adesão muito aquém deste valor, pouco menor do que o encontrado também no Brasil, no Estado de São Paulo 5 .

Apesar da disponibilidade dos ARV no País, os resultados de não adesão e de recusa ao tratamento anti-retroviral indicam problemas de natureza diversa. Considerando que a populaçãa estudada era virgem de tratamento ARV, o fato de que cerca de $52,4 \%$ dos casos já tenham chegado a um serviço especializado com algum sintoma, sinal ou doença indicativa de imunodeficiência (Tabela 4) sugere que os indivíduos com infecção HIV no Rio Grande do Norte estão sendo diagnosticados e tratados muito tardiamente. Outros resultados, como a maioria dos casos com indicação de terapia ARV só tomarem conhecimento da infecção pelo HIV após ingresso em serviço especializado $(59,4 \%)$ e o fato de mais de $67 \%$ dos casos terem contagem de linfócitos TCD4+ abaixo de 200/ml (Tabela 2) reforçam a hipótese de acesso tardio.

Esses achados corroboram dados de relatório oficial do Ministério da Saúde que inclui, como um dos principais desafios para os gestores do sistema de saúde, a necessidade de ampliação das modalidades de assistência não convencional, com vistas a aumentar o acesso a grupos potencialmente expostos à infecção pelo HIV, ou em situação de maior vulnerabilidade, assim como a descentralização da rede de assistência para municípios do interior ${ }^{23}$. Essas medidas possibilitarão a oportunidade de diagnóstico precoce da infecção pelo HIV e o início de tratamento na fase assintomática da doença, além de permitir orientações sobre prevenção da transmissão do vírus.

Para que sejam verificados os benefícios da terapia anti-retroviral tais como melhora na condição física e emocional; reduçãa do risco de desenvolver infecções oportunistas; e aumento na sobrevida, entre outros, decorrentes da redução da supressão viral causada pelos medicamentos - os indivíduos com recomendação de tratamento devem estar suficientemente informados sobre a sua doença e a importância da regularidade do tratamento, a fim de evitar resistência viral e falha terapêutica. A falta de obediência às instruções médicas adiciona um problema para os serviços de saúde, que necessitam criar mecanismos de acompanhamento para identificar os principais fatores relacionados à não adesão aos medicamentos e, também, ao adiamento ou recusa da prescrição inicial.

Para alguns pesquisadores, a aceitação e a adesão aos ARV estão significativamente associadas ao esquema de medicamentos, à confiança depositada no sistema de saúde e na relaçãa interpessoal com os profissionais de saúde ${ }^{24,25}$. O papel do serviço de saúde, principalmente pela capacidade de diálogo e de negociação, é extremamente importante na superação das dificuldades relacionadas ao tratamento ${ }^{5}$.

$\mathrm{O}$ achado da presente investigação, de que cerca de $10 \%$ dos pacientes com indicação médica para iniciar um dos esquemas de terapia ARV não compareceram para receber a medicação nos seis primeiros meses após a prescrição, indica a necessidade da realização de estudos que levem em conta as diversidades regionais e de assistência no País, de modo a dimensionar esta questão no nível nacional, e suas implicações nas políticas de aquisiçãa e distribuiçã̃o de ARV no Brasil. Além disso, este achado considerado recusa ao tratamento ARV aponta, igualmente, na direção da realização de estudos quanti-qualitativos que abordem a questão do adiamento ou da recusa do cumprimento da prescrição inicial de anti-retrovirais à luz do processo de aceitação pelo indivíduo de sua doença e dos benefícios da utilização correta dos medicamentos. Estudo qualitativo realizado no Brasil sobre a utilização de medicamentos por indivíduos portadores do HIV identificou, como primeiro problema, a recusa ao uso de ARV. Segundo os autores, embora a terapia ARV tenha sido prescrita, o portador do HIV adia ou rejeita o cumprimento da prescrição; em alguns casos havia referência ao início de uso até três anos após a prescriçãa ${ }^{26}$. Ainda sobre o resultado da recusa, chama a atenção o grande percentual de recusa em mulheres gestantes com indicação de terapia ARV. Trata-se de achado relevante, uma vez que a recusa ao uso da terapia ARV em gestantes com infecção pelo HIV traz implicações diretas sobre o risco de transmissão vertical do HIV, e o conseqüente aumento de casos de AIDS em crianças. A possibilidade de redução significativa da transmissão vertical do HIV durante a gestação, o parto e a amamentação, com o uso de AZT pela mãe, já foi demonstrada pelo Protocolo 076 do AIDS Clinical Trial Group, em 1994. Desde então, em todo o mundo, esforços têm sido feitos para a implementaçãa de ações voltadas para a prevenção da transmissão do HIV da mãe infectada para seu filho, com resultados bastante animadores ${ }^{27}$.

No presente trabalho, índice baseado na freqüência de visitas agendadas à farmácia de dispensação dos medicamentos ARV foi utilizado para avaliar um dos aspectos dos pacientes em relação ao tratamento. Trata-se de um modo indireto e de fácil operacionalização para avaliar a chamada interrupção ao tratamento. Por razões óbvias, implica uma sub-estimação do nível de adesão, porém de grau não muito elevado. Uma das limitações deste procedimento é que esta medida não permite afirmar se a medicação foi utilizada na quantidade prescrita e/ou nos horários definidos. Contudo, é razoável admitir que um paciente que acata uma recomendação médica e comparece regularmente à unidade de assistência farmacêutica está fazendo uso adequado da medicação. O comparecimento às datas agendadas na 
farmácia pode produzir bons indicadores da adesão real e constituir um método útil e rápido na identificação de indivíduos em risco de não adesão, com possível acompanhamento pelo serviço de assistência.

Em relação aos fatores associados à adesão, algumas investigações mostraram que as baixas condições socioeconômicas dos doentes influenciaram decisivamente na não-adesão ao tratamento antiretrovira ${ }^{5,10}$, enquanto em outros não se estabeleceu essa associação $0^{28,29}$. A divergência dos resultados pode ser, em grande parte, explicada pelas diferenças no padrão socioeconômico entre os grupos pesquisados. $O$ resultado da presente pesquisa revela uma associação estatisticamente significativa entre a interrupção ao tratamento antiretroviral e indivíduos com escolaridade elementar (analfabeto ou até três anos), aqui considerado como indicador de baixo nível socioeconômico. Em relação à idade, o achado de associação estatisticamente significativa entre a interrupção e indivíduos jovens corrobora resultados obtidos por outros pesquisadores ${ }^{30}$.

As características relacionadas à doença, como a presença de sintomas quando do início da terapia anti-retroviral e o valor da contagem dos linfócitos T CD4+, não se mostraram associadas à interrupção. Por outro lado, o presente trabalho revelou associação entre a interrupção e o fato de o doente ter tido indicação de uso de terapia anti-retroviral após internação hospitalar. Não parece haver consenso na literatura sobre questões relacionadas à gravidade da doença e a adesão $0^{5}$.

tipo de esquema de anti-retrovirais não se associou à interrupção, na presente investigação. Por comparação com outras doenças, o regime terapêutico com os ARV tem sido muito lembrado por vários autores como fator potencialmente associado à não adesão, mas os estudos empíricos ainda são raros e controversos. Entre os motivos identificados para a não adesão, cita-se: tomar mais de três doses por dia, tamanho do comprimido e restrição dietética ${ }^{25,31}$. Pesquisas mais recentes têm mostrado, porém, que o número de medicamentos e de doses não influenciam a adesão ${ }^{29,32}$.

As variáveis mais consistentemente associadas à interrupção foram o uso de drogas lícitas ou ilícitas e a história de tratamento psiquiátrico anterior ao diagnóstico da AIDS. Estes achados corroboram os resultados de outros trabalhos ${ }^{18,33}$, inclusive aqueles relacionados ao uso de outros medicamentos além dos anti-retrovirais ${ }^{4}$.

\section{CONCLUSÃo}

Os resultados sugerem que a interrupção deve ser um problema crítico durante os seis primeiros meses seguintes ao início da terapia anti-retroviral em indivíduos virgens de tratamento, e, especialmente, entre os que têm história de tratamento psiquiátrico; usam ou fizeram uso de drogas lícitas ou ilícitas até um ano antes de iniciar os ARV; iniciam a terapia ARV após internamento hospitalar; têm baixo nível de escolaridade; e estão no grupo de 25 a 34 anos de idade.

\section{Conflito de interesse: não há.}

\section{Summary}

Cofactors of antiretroviral treatment interruption IN Cases of adults with AIDS. Rio Grande do Norte, Brazil, 1999-2002
BACKGROUND. The purpose of this study is to determine factors associated to the interruption of antiretroviral treatment in adults with AIDS in the State of Rio Grande do Norte, Brazil.

METHODS. This was a population-based study, using data from the State's sources of vital statistics. Interruption was calculated using data on the number of programmed visits to the pharmacies, taking into account the date of the first prescription. It was considered that patients had adhered to the treatment if they came to at least $80 \%$ of the programmedvisits.

RESULTS. The overall percentage for non interruption of the antiretroviral therapy was 64.1\%. No association was found with the following: gender, type of exposure, residence, nor with the type of antiretroviral combination prescribed. After multivariate analysis, significant associations continued to be found between interruption and inhospital stay, use of drugs, psychiatric treatment, low level of education and age ranging from 25 to 34 years.

CONCLUSIONS. These results point towards significant associations between interruption of antiretroviral treatment and the beginning of antiretroviral therapy during the in-hospital stay, the use of legal or illegal drugs, a history of psychiatric treatment, low level of education, and age ranging from 25 to 34 years. [Rev Assoc Med Bras 2006; 52(2): 86-92]

KEY WORDS: AIDS. Antiretroviral therapy. Interruption of treatment. Adherence.

\section{REFERÊnCIAS}

I. Eraker SA, Kirscht JP, Becker MH. Understand and improving patient compliance. Ann Intern Med 1984; 100:258-68.

2. Stephenson J. AIDS researchers target poor adherence. JAMA 1999; 281:1069.

3. Haynes RB, McKibbon KA, Kanani R. Systematic review of randomised trials of interventions to assist patients to follow prescriptions for medications. Lancet 1996;348:383-6

4. Lerner BH, Gulick RM, Dubler NN. Rethinking No-adherence: Historical Perspectives on Triple-Drug Therapy for HIV Disease. Ann Intern Med 1998; 129:573-8.

5. Nemes MIB, Souza MFM, Kalichman AO, Grangeiro A, Souza RA, Lopes JF. Prevalência da aderência e fatores associados. In: Coordenação Nacional de DST e AIDS, Série avaliação: Aderência ao tratamento por anti-retrovirais. Brasília: Ministério da Saúde; 2000.

6. Haubrich RH, Little SJ, Currier JS, Forthal DN, Kemper CA, Beall GN, et al,. The value of patient-reported adherence to antiretroviral therapy in predicting virologic and immunologic response. California Collaborative Treatment Group. AIDS 1999; 13:1099-107.

7. Hetch FM. Measuring HIV treatment adherence in clinical practice. AIDS Clin Care 1998; 10:57-9.

8. Clough LA, D'Agata E, Raffanti S. Factors that predict incomplete virological response to protease inhibitor-based antiretroviral therapy. Clin Infect Dis 1999; 29:75-81.

9. Carpenter CCJ, Cooper DA, Fischl MA, Gatell JM, Gazzard BG, Hammer SM, et al. Antiretroviral therapy in adults. Updated Recommendations of the International AIDS Society - USA Panel. JAMA 2000; 283:38I-90.

I0. Lucas GM, Chaisson RE, Moore RD. Highly active antiretroviral therapy in a large urban clinic: risk factors for virologic failure and adverse drugs reactions. Ann Intern Med 1999; 131:81-7.

I I. Maher K, Klimas N, Fletcher MA, Cohen V, Maggio M, Triplett J, et al. Disease therapy progression, adherence, and response to protease inhibitor for hiv infection in an urban veterans affairs medical center. J Acquir Immune Defic Syndr 1999;22: I-20.

12. Eldred L. Adherence in the era of protease inhibitors. John Hopkins AIDS 
BRTTO AM ET AL.

Service 1998 [on line]. Available from: http://www.hopkins-AIDS.edu/ jhas_htmlcode/jhas_outres/jhas_outres_therapy.html. [Cited 200 I May 7].

13. Walsh J, Dalton M. Gill J, Wilkinson D, Burgess AP, Gazzard BG. Adherence to proteinase inhibitor based highly effective antiretroviral therapy (HAART). In: I 2th World AIDS Conference, Geneva; 1998. Abstracts n. 12298

14. Holodniy M. What is antiretroviral failure? [Editorial response]. Clin Infect Dis 1999:29:82-4

15. Louie JK, Hsu LC, Osmond DH, Katz MH, Schwarcz SK. Trends in causes of death among persons with acquired immunodeficiency syndrome in the era of highly active antiretroviral therapy, San Francisco, 1994-1998. J Infect Dis 2002; 1 86: 1023-7.

16. Carr A, Cooper DA. Gap between biology and reality in AIDS. Lancet 1998; 352 (Suppl 4): 16.

17. Centers for Disease Control and Prevention. Guidelines for the use of anti-retroviral agents in HIV-Infected Adults and Adolescent. MMWR Morb Mortal Wkly Rep 1999:48(RR-5): I -49.

18. Bomtempo NM. Estudo de fatores de risco para uso irregular do tratamento anti-retroviral, em um serviço público de referência, em Minas Gerais, 1998-1999 [dissertação]. Belo Horizonte: Faculdade de Medicina, Universidade Federal de Minas Gerais; 2000.

19. Roberts KJ. Barriers to and facilitators of HIV-patient adherence to antiretroviral treatment regimens. AIDS Patient Care STDS 2000; 14:155-68.

20. Ministério da Saúde. Coordenação Nacional de Doenças Sexualmente Transmissíveis e AIDS. Bol Epidemiol AIDS 2002; I 5(2).

21. Ministério da Saúde. Coordenação Nacional de Doenças Sexualmente Transmissíveis e AIDS. Recomendações para terapia anti-retroviral em adultos e adolescentes infectados pelo HIV - 2000. Brasília; 2000.

22. Ministério da Saúde. Coordenação Nacional de Doenças Sexualmente Transmissíveis e AIDS. Recomendações para terapia anti-retroviral em adultos e adolescentes infectados pelo HIV - 200 I. Brasília; 200 I.

23. Ministério da Saúde. Coordenação Nacional de Doenças Sexualmente Transmissíveis e AIDS. Terapia anti-retroviral e Saúde Pública: Um balanço da experiência brasileira. Brasília; 1999.

24. Chesney MA. Factors affecting adherence to antiretroviral theraphy. $\mathrm{Cl}$ Infect Dis 2000; 30(Suppl 2): 17 |-6.
25. Nieuwkerk P, Gisolf EH, Van Leeuwen R, Danner SA, De Boer IB, Sprangers MAG. Self-reported adherence to ritonavir/saquinavir(RTV/ SQV) and ritonavir/saquinavir/stavudine (RTV/SQV/d4T) in a randomized clinical trial: preliminary results. In: 12th World AIDS Conference, Geneva; 1998. Abstracts n.32362.

26. Acúrcio FA, Guimarães MDC. Utilização de medicamentos por indivíduos HIV positivos: abordagem qualitativa. Rev Saúde Pública 1999;33:73-84.

27. Lindegren ML, Byers RH Jr., Thomas P, Davis SF, Caldwell B, Rogers M, et al. Trends in perinatal transmission of HIV/AIDS in the United States. JAMA 1999; 282:531-8.

28. Corden ZM, Bosley CM, Rees PJ, Cochrane GM. Home nebulized therapy for patients with COPD: patient compliance with treatment and its relation to quality of life. Chest | $997 ;$ | | 2: | 278-82.

29. Wood E, Montaner JSG, Chan K, Tyndall MW, Schechter MT, Bangsberg $D$, et al. Socioeconomic status, acess to triple theraphy, and survival from HIV-disease since 1996. AIDS 2002; 16:2065-72.

30. Becker SL, Dezii CM, Burtcel B, Kawabata H, Hodder S. Young HIVinfected adults are at greater risk for medication nonadherence. Medscape General Medicine 2002; 4(3):6. Available from: http://www.medscape. com/viewarticle. [Cited 2003 Jan I0]

31. Figueiredo RM, Sinkoc VM, Gallani MCB, Tomazin C, Cavinatto SM, Assumpcao ISA. Adherence of patients with AIDS to treatment with antiretroviral medications: Difficulties related and proposition of attenuating measures. In: 12th World AIDS Conference, Geneva; 1998. Aabstracts n.42442.

32. Wilson IB, Tchetgen E, Spiegelman D. Patterns of adherence with antiretroviral medications: An examination of between-medication differences. J Acquir Immune Defic Syndr 200 I;28:259-63.

33. Howard AA, Arnsten JH, Lo, Y, Vlahov D, Rich JD, Schuman P, et al. A prospective study of adherence and viral load in a large multi-center cohort of HIV-infected women. AIDS 2002; 16:2175-82.

Artigo recebido: 17/1 1/04

Aceito para publicação: 06/12/05 